\title{
Detection of isoprene traces in exhaled breath by using photonic crystals as a biomarker for chronic liver fibrosis disease
}

\section{Ahmed Mehaney}

Beni Suef University Faculty of Science

Hussein A. Elsayed ( $\nabla$ drhussien85sc@gmail.com )

Beni Suef University Faculty of Science https://orcid.org/0000-0001-7819-9476

Ashour M. Ahmed

Beni Suef University Faculty of Science

\section{Research Article}

Keywords: Isoprene, Photonic crystals, Liver fibrosis disease, Dry exhaled breath, Tamm resonance, Biomarker.

Posted Date: April 20th, 2021

DOI: https://doi.org/10.21203/rs.3.rs-443784/v1

License: (c) (1) This work is licensed under a Creative Commons Attribution 4.0 International License. Read Full License 


\title{
Detection of isoprene traces in exhaled breath by using photonic crystals as a
}

\section{biomarker for chronic liver fibrosis disease}

\author{
Ahmed Mehaney ${ }^{1}$, Hussein A. Elsayed ${ }^{1, \$}$ and Ashour M. Ahmed ${ }^{2}$ \\ ${ }^{1}$ TH-PPM Group, Physics Department, Faculty of Science, Beni-Suef University, Beni-Suef, \\ 62512, Egypt \\ ${ }^{2}$ Physics Department, Faculty of Science, Beni-Suef University, Beni-Suef, 62512, Egypt \\ \$Correspondind Author : Hussein A. Elsayed - E-mails: drhussien85sc@gmail.com, \\ hussien.abdelghani@science.bsu.edu.eg.
}

\begin{abstract}
Detection of blood-carried volatile organic compounds (VOCs) existing in the exhaled breath of human is an attractive research point for noninvasive diagnosis of diseases. In this research, we introduce a novel application of photonic crystals (PCs) for the detection of isoprene traces in the exhaled breath as a biomarker for liver fibrosis. This idea is introduced for the first time according to the best of our knowledge. The proposed sensor structure is a one-dimensional (1D) PC constructed from a multilayer stack of two dielectric materials covered with an air cavity layer filled with the dry exhaled breath (DEB) and a thin metallic layer of $\mathrm{Au}$ is attached on the top surface. Hence, the proposed sensor is configured as, [prism/Au/air cavity/(GaN/SiO 2$\left.)^{10}\right]$. The transfer matrix method and the Drude model are adopted to calculate the numerical simulations and reflection spectra of the design. The essential key for sensing isoprene levels is the resonant optical Tamm plasmon (TP) states within the photonic bandgap. The obtained numerical results are promising such as high sensitivity (S) of $0.321 \mathrm{~nm} / \mathrm{ppm}$ or 278720 $\mathrm{nm} / \mathrm{RIU}$. This technique can be reducing the risk of infection during the taking of blood samples by syringe. Also, it can prevent the pain of patients. Finally, this
\end{abstract}


work opens the door for the detection of many diseases by analyzing the breaths of patients based on photonic crystals.

\section{Keywords:}

Isoprene; Photonic crystals; Liver fibrosis disease; Dry exhaled breath; Tamm resonance; Biomarker.

\section{Introduction}

In recent years, exhaled human breath analysis became an urgent tool for the detection of various diseases and assists clinicians in early diagnose of the impending effects of diseases [1]. The actual beginning for monitoring diseases based on exhaled breath analysis was dedicated by Linus Pauling [2]. However, breath analysis was an ancient diagnosis tool since Hippocrates learned his students to utilize exhaled for identifying many diseases such as failing kidneys and liver disease [3]. From this date, the exhaled breath analysis techniques attracted researchers' attention because they considered as a specific diagnosis of diseases, noninvasive, and inexpensive [1,4].

Previous studies proved that healthy people exhaled about 874 types of volatile organic compounds (VOCs) such as acetone (1.2 - $900 \mathrm{ppb})$, isoprene (12-580 ppb), methanol (160 - 2,000 ppb), and ethanol (13 1,000 ppb) [2- 4]. Therefore, any disorder in the levels of exogenous VOCs could reflect the physiological state of the person. For example, increasing the percentages of isoprene molecules in the exhaled breath of a person can be an indicator of liver fibrosis disease [5], acetonitrile level increase in the exhaled breath of smokers, and high concentrations of acetone represent diabetes disease [6]. Consequently, many techniques were presented for the diagnosis of diseases depending on the concentrations and percentages of VOCs in exhaled breath. For example, Popa et 
al. investigated that high levels of exogenous ammonia and ethylene in human breath is a diagnosis of schizophrenia [7]. Also, lung cancer can be diagnosed based on levels of exogenous toluene as reported by Szulejko et al. [8].

More recently, optical and surface plasmon resonance (SPR)-based sensors have introduced novel results in the applications of biosensors and biomarkers based on levels of VOCs in patient respiration [9-11]. Optical biosensors are characterized by high performance, inexpensive, and compact design which can be inserted with a lab-on-a-chip device $[12,13]$. Photonic crystals (PCs) are considered fascinating optical sensors and biosensors in recent years [14- 16]. PCs are periodic structures in $1 \mathrm{D}, 2 \mathrm{D}$, or $3 \mathrm{D}$ designs that able to control the propagation of electromagnetic waves (E.M waves) within ranges of frequencies called photonic band gaps (PnBG). The formation of PnBG due to the refractive index contrast between the constituent materials [17- 19]. Making a cavity inside the periodic design of PCs leads to the generation of local resonance modes that were used in gas or liquid sensor applications. Clevenson et al. reported a 1D PC as a gas sensor with low concentrations (ranging from 600 parts per million (ppm)) [20]. The temperature and concentration of ethylene/air mixture were measured by a 1D PC sensor presented by Chen et al. [21]. A 2D PC gas sensor with a high sensitivity of 575 $\mathrm{nm} / \mathrm{RIU}$ was introduced by Anamoradi et al. [22].

Another way for producing resonant modes in PCs is achieved based on surface plasmon resonance (SPR) and optical Tamm plasmon (TP) resonance. TP resonance is defined as the quantization of free electrons oscillations at a metaldielectric interface [23]. A theoretical 1D PC sensor was presented based on TP resonance. It provided higher sensitivity than conventional PC sensors that reached to $5018 \mathrm{~nm} / \mathrm{RIU}$ [13]. Also, the 1D PC sensor was investigated experimentally by Auguie et al., who obtained a sensitivity of $55 \mathrm{~mm} / \mathrm{RIU}$ [24]. 
On the other side, about 2 million deaths per year worldwide due to liver disease. The eleventh most common cause of death worldwide is liver fibrosis. The fibrosis making it hard for the liver to function and it is life-threatening. Liver fibrosis is caused by many forms of liver diseases such as chronic alcoholism and viral hepatitis (hepatitis B, C, and D). Egypt has the highest prevalence of hepatitis C virus infection in the world. The early diagnosis may prevent damage from occurring in the liver. Hence, this present study focuses on the application of exhaled isoprene sensing as a possible and advanced tool to diagnose liver fibrosis disease.

For this purpose, a novel application of 1D PCs as a biomarker or biosensor for sensing the lowest isoprene levels in the dry exhaled breath based on the phenomenon of TP resonance is presented in this work. The 1D PC gas sensor is designed as a multilayer from two dielectric materials with a thin metal layer on its face. Then, the exhaled breath with different levels of isoprene (from $0 \mathrm{ppm}$ to 100 ppm) is proposed to pass through an air cavity between the multilayer stack and the metal layer. The well-known transfer matrix method (TMM) was used to manipulate the propagation of EM waves through the sensor structure and calculate the reflection spectra of each level of exhaled isoprene. Moreover, the most important performance parameters for any sensor such as quality factor, sensitivity, detection limit, the figure of merit, and sensor resolution were calculated for each isoprene level.

\section{Sensor design and numerical analysis}

\subsection{Sensor design}

The suggested design of the 1D PC gas sensor is shown in the schematic diagram in Fig. 1. Firstly, the sensor structure is a periodic multilayer 1D PC from $\mathrm{GaN} / \mathrm{SiO}_{2}$. Secondly, a thin metals layer $(\mathrm{Au})$ is attached to the front of the 
$\mathrm{GaN} / \mathrm{SiO}_{2}$ multilayer stack. Thirdly, the exhaled breath with the different isoprene levels should pass and fill an air cavity between the metal layer and the PC multilayer. The thickness of the multilayer stack is proposed to be $a=d_{1}+d_{2}$, where, $d_{1}$ and $d_{2}$ are the thickness of $\mathrm{GaN}$ and $\mathrm{SiO}_{2}$, respectively. Also, the thickness of $\mathrm{Au}$ and the cavity layer is set to be $\mathrm{d}_{\mathrm{m}}$ and $\mathrm{d}_{\mathrm{c}}$, respectively. Finally, a prism is set on the metal layer to enhance the reflection for the incident EM on the PC design [16]. Consequently, the complete design of the PC gas sensor can be configured as the following, [prism/Au/air cavity $/\left(\mathrm{GaN} / \mathrm{SiO}_{2}\right)^{\mathrm{N}}$ ]. Where $\mathrm{N}$ is the unit cells (periods) number. The choosing of $\mathrm{GaN}$ and $\mathrm{SiO}_{2}$ due to their excellent optical, chemical stability, and mechanical properties $[25,26]$. Also, they are used widely in the design of PCs sensors theoretically and experimentally [13, 15, 16 ,19]. 


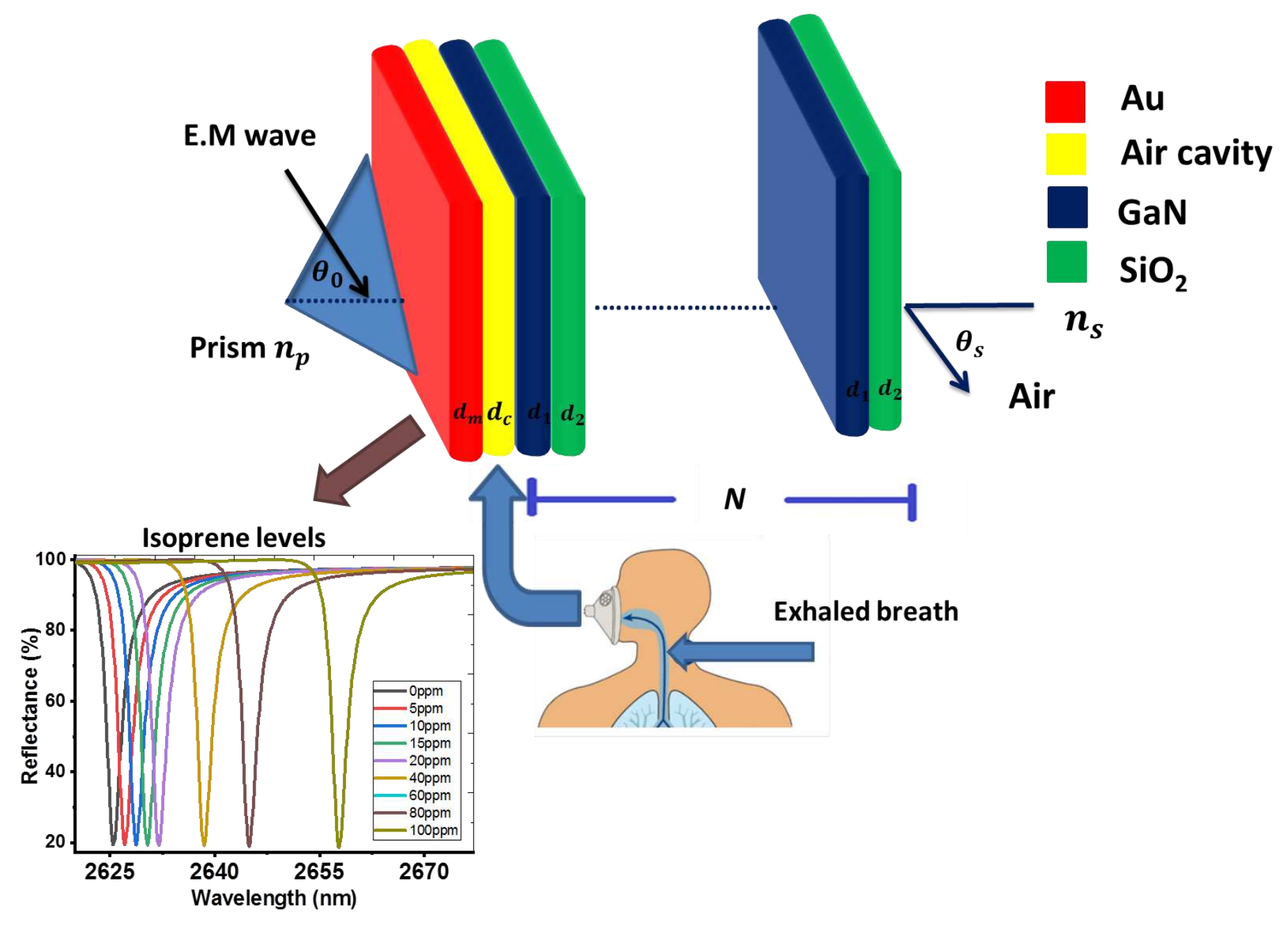

Fig. 1. The schematic diagram of the proposed sensor design [prism/Au/air cavity/(GaN/SiO 2$\left.)^{N}\right]$.

\subsection{Analysis of the transfer matrix method}

Here, we intend to investigate the basic equations implemented to obtain the optical reflectance of the incident EM waves through the proposed sensor design. The propagation of EM waves through periodic structures is manipulated by using the transfer matrix method (TMM) [15]. TMM is considered an accurate method in studying and manipulating the propagation of various types of waves through the 1D periodic lattices [15-17]. The TMM is completely dependent on Maxwell's 
equations and their solution through each layer (j) or repeated unit cells of the lattice constant (a).

As shown in Fig. 1, the incident EM wave is assumed to propagate through $x$ direction. Therefore, the two polarization modes (electric and magnetic fields) are oscillating through the $(y-z)$ plane. For the transverse electric (TE) mode, the general solution of the incident EM wave through the $\mathrm{j}^{\text {th }}$ layer can be written as,

$$
\begin{aligned}
& \hat{E}_{j}(y, x)=E_{j}(x) \exp \left[i\left(k_{y} y-\omega t\right)\right] \hat{y}, \\
& \hat{H}_{j}(z, x)=H_{j}(x) \exp \left[i\left(k_{y} y-\omega t\right)\right] \hat{z},
\end{aligned}
$$

Where, $\mathrm{k}_{\mathrm{y}}$ is the wavevector, $\omega$ is the angular frequency, $\mathrm{E}(x)$ and $\mathrm{H}(x)$ are the components of the electric and magnetic fields, respectively. They can be written within a specific layer $j^{\text {th }}$ as,

$$
\begin{aligned}
& E_{j}(x)=S_{j} \exp \left(-i k_{j} x\right)+P_{j} \exp \left(i k_{j} x\right), \\
& H_{j}(x)=n_{j}\left[-S_{j} \exp \left(-i k_{j} x\right)+P_{j} \exp \left(i k_{j} x\right)\right], \\
& k_{j}=(2 \pi / \lambda) n_{j} .
\end{aligned}
$$

Where, $k_{j}$ is the wavenumber in layer $j$ and $n_{j}$ defines its refractive index. $S_{j}$ and $P_{j}$ are the electric and magnetic field amplitudes, respectively. By substituting equation (1) in equation (2), the following matrix form can be obtained as,

$$
\left(\begin{array}{c}
E_{j}(x) \\
H_{j}(x)
\end{array}\right)=\left(\begin{array}{cc}
\exp \left(-i k_{j} x\right) & \exp \left(i k_{j} x\right) \\
-n_{j} \exp \left(-i k_{j} x\right) & n_{j} \exp \left(i k_{j} x\right)
\end{array}\right)\left(\begin{array}{c}
S_{j} \\
P_{j}
\end{array}\right)
$$

TMM requires the continuity of the EM wave solution through the boundaries between each two successive layers $j$ and $(j+1)$. Therefore, the elements of the $E_{j}$ (x) and $\mathrm{H}_{\mathrm{j}}(\mathrm{x})$ elements at these boundaries are given as, 


$$
\begin{aligned}
& \left(\begin{array}{l}
E_{j}(x) \\
H_{j}(x)
\end{array}\right)= \\
& \left(\begin{array}{cc}
\frac{\left[\exp \left(-i k_{j} \chi_{j}\right)+\exp \left(i k_{j} \chi_{j}\right)\right]}{2} & {\left[\frac{\exp \left(-i k_{j} \chi_{j}\right)-\exp \left(i k_{j} \chi_{j}\right)}{2 \tau_{j}}\right]} \\
\frac{\tau_{j}\left[\exp \left(-i k_{j} \chi_{j}\right)-\exp \left(i k_{j} \chi_{j}\right)\right]}{2} & \frac{\left[\exp \left(-i k_{j} \chi_{j}\right)+\exp \left(i k_{j} \chi_{j}\right)\right]}{2}
\end{array}\right)\left(\begin{array}{l}
E_{j+1}(x) \\
H_{j+1}(x)
\end{array}\right) .
\end{aligned}
$$

Equation (4) can be rewritten in a simple form as,

$$
\left(\begin{array}{c}
E_{j}(x) \\
H_{j}(x)
\end{array}\right)=\left(\begin{array}{cc}
\cos \left(k_{j} \chi_{j}\right) & -i \sin \left(k_{j} \chi_{j}\right) / \tau_{j} \\
-i \tau_{j} \sin \left(k_{j} \chi_{j}\right) & \cos \left(k_{j} \chi_{j}\right)
\end{array}\right)\left(\begin{array}{c}
E_{j+1}(x) \\
H_{j+1}(x)
\end{array}\right)=m_{j}\left(\begin{array}{c}
E_{j+1}(x) \\
H_{j+1}(x)
\end{array}\right) .
$$

Where, $\theta_{j}$ is the angle of incidence through the layer $j$ th, $\chi_{j}$ and $\tau_{j}$ are two coefficients with the values, $\chi_{j}=d_{j} \cos \theta_{j}, \tau_{j}=\left(\sqrt{\varepsilon_{0} / \mu_{0}}\right) n_{j} \cos \theta_{j}$, where, $\mathrm{d}_{\mathrm{j}}$ the thickness of the layer jth and $\varepsilon_{0}$ and $\mu_{0}$ are the permittivity and permeability of a vacuum. After few steps can be found in references [27, 28], we could deduce the total electric and magnetic field components at the boundaries between each two successive layers as a $2 \times 2$ transfer matrix $\mathrm{M}$ as,

$$
M=\left(\begin{array}{ll}
M_{11} & M_{12} \\
M_{21} & M_{22}
\end{array}\right)=m_{m} m_{c}\left(m_{1} m_{2}\right)^{N}
$$

Where, the elements $m_{m}, m_{c}, m_{1}$, and $m_{2}$ of the transfer matrix $M$ represent the solution of the EM propagation in the Au layer, cavity air, and binary multilayer stack $\left(\mathrm{GaN}, \mathrm{SiO}_{2}\right)$, respectively. Now, we could write the reflectance coefficient as: 


$$
r=\frac{\left(M_{11}+M_{12} \zeta_{s}\right) \zeta_{0}-\left(M_{21}+M_{22} \zeta_{s}\right)}{\left(M_{11}+M_{12} \zeta_{s}\right) \zeta_{0}+\left(M_{21}+M_{22} \zeta_{s}\right)}
$$

where, the coefficient $\zeta_{0(S)}=\left(\sqrt{\varepsilon_{0} / \mu_{0}}\right) n_{0(S)} \cos \theta_{0(S)}$, the subscripts 0 and $\mathrm{S}$ refer to the incidence (prism) and substrate (air) media, respectively. Finally, the reflectance $(\mathrm{R})$ of the incident EM waves on the 1D PC design can be investigated as follows $[17,27]$;

$$
R=\left|r^{2}\right|
$$

Similarly, the matrix analysis of the TM mode can be deduced using equations (5-8) but with $\tau_{j}=\left(\sqrt{\varepsilon_{0} / \mu_{0}}\right) \cos \theta_{j} / n_{j}$.

\subsection{Materials refractive index}

As it well-known, metals have a high absorption for the incident EM waves, so its dielectric constant is a complex function. The complex dielectric constant $\left(\epsilon_{\mathrm{m}}\right)$ of any metal is given according to Drude model as the following equation [29],

$$
\epsilon_{\mathrm{m}}=\epsilon_{1}+\mathrm{i} \epsilon_{2}=1-\frac{\omega_{\mathrm{p}}^{2}}{\omega(\omega+\mathrm{i} \gamma)} .
$$

Where, $\epsilon_{1}$ and $\epsilon_{2}$ represent the real and imaginary parts of the complex dielectric constant, respectively. Also, $\omega_{p}$ is the plasma frequency of metal (here is $\mathrm{Au}$ ) and $\gamma$ is the damping constant. Then, the refractive index can be written as the square root of the complex dielectric constant, $\mathrm{n}_{\mathrm{m}}=\sqrt{\epsilon_{\mathrm{m}}}$.

Concerning the refractive index of $\mathrm{SiO}_{2}$ is a wavelength-dependent as the following equation [30]:

$$
n_{\mathrm{SiO}_{2}}(\lambda)=1.4513+0.0026 \lambda-0.0045 \lambda^{2} \text {. }
$$


Where, $\lambda$ (in microns) is the wavelength of the incident E.M wave. Also, the refractive index of $\mathrm{GaN}$ is a wavelength-dependent as well and it is given as [31]:

$$
n_{G a N}^{2}(\lambda)-1=2.60+\frac{1.75 \lambda^{2}}{\lambda^{2}-(0.256)^{2}}+\frac{4.1 \lambda^{2}}{\lambda^{2}-(17.86)^{2}}
$$

\section{Numerical results and discussions}

We propose here the materials parameters and present the numerical investigations concerning the interaction between the incident EM waves and the sensor design at different levels of isoprene.

For this purpose, the GaN layer is set with a thickness $d_{1}=200 \mathrm{~nm}$ while the $\mathrm{SiO}_{2}$ layer is set with a thickness $\mathrm{d}_{2}=900 \mathrm{~nm}$. The number of periods is proposed to be $\mathrm{N}=10$, the angle of incidence is taken as $\theta_{0}=50^{\circ}$ and the prism is assumed with the index $n_{p}=1.3$. The metallic layer of $A u$ and air cavity layer are designed with thicknesses $d_{m}=10 \mathrm{~nm}$ and $d_{c}=14 \mu \mathrm{m}$, respectively. Also, the operating wavelength of the incident EM waves will be in the range of $2000 \mathrm{~nm}$ to $4000 \mathrm{~nm}$. All the aforementioned parameters were taken to achieve a higher sensor performance and a wide bandgap after multi optimization steps.

In the beginning, we will investigate the reflection properties of the PC multilayer stack in the absence of the metal layer and air cavity in order to set the best boundary conditions of the PnBG. Therefore, the reflectance of the 1D PC structure that is arranged as, $\left[\right.$ prism $\left./\left(\mathrm{GaN} / \mathrm{SiO}_{2}\right)^{10}\right]$ is shown in Fig. 2. As shown in this figure, a wide perfect photonic bandgap appears in the wavelength range $2337.5 \mathrm{~nm}-3347.7 \mathrm{~nm}$ and with a bandwidth of $1010.2 \mathrm{~nm}$. The bandgap is characterized by nearly zero transmission of EM waves. Such a wide bandgap is due to the high mismatch in refractive index between $\mathrm{GaN}$ and $\mathrm{SiO}_{2}$ materials, and it is resulted due to the destructive interference of EM waves at layers boundaries $[13,15]$. The production of wide band gaps in the refection spectrum of PCs is 
considered a desired indicator for designing sensor platforms. This is facilities the localization of resonant modes within the band gap without crosstalk with other ripples or reflected dips.

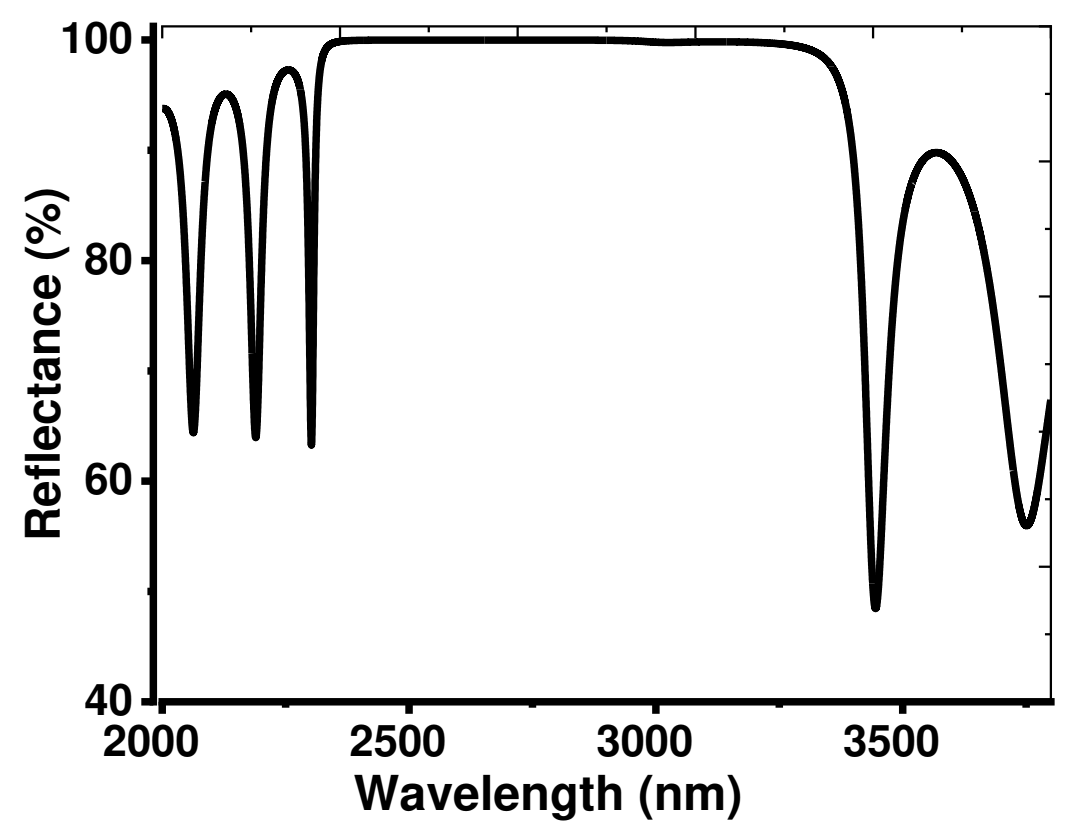

Fig. 2. The reflectance of the $1 D$ PC gas sensor that constructed as $\operatorname{prism} /\left(\mathrm{GaN}_{\mathrm{SiO}}\right)^{10} /$ air.

By embedding a cavity layer of thickness $14 \mu \mathrm{m}$ in front of the previous PC $\mathrm{GaN} / \mathrm{SiO}_{2}$ multilayer, the designed structure is considered as prism/air cavity $/\left(\mathrm{GaN} / \mathrm{SiO}_{2}\right)^{10} /$ air. As seen in Fig. 3, the reflectance intensity of this structure is increased due to the presence of the air cavity layer. Also, the PnBG width became wider due to the insertion of the air cavity layer, since, the refractive index mismatch becomes higher in that case, prsim, air cavity and GaN layer. The wavelength range of that bandgap extended from 2220.5 to $3398.9 \mathrm{~nm}$ with a bandwidth of $1178 \mathrm{~nm}$. Moreover, more ripples with a high reflection intensity are produced outside the bandgap due to the insertion of that defect air cavity layer. 
Where, the large thickness of the air cavity layer $(14 \mu \mathrm{m})$ is another reason for producing these large number of ripples. In addition to that, the most interesting defect dip here is the ones that localized at the wavelength of $3500 \mathrm{~nm}$. This defect dip is produced exactly according to the well-known quarter wave stack ( $\lambda_{\text {res }}=$ $\frac{n d}{4}$ ), where, $\lambda_{\text {res }}$ is the dip wavelength, $n$ is the air cavity refractive index ( here $n=$ 1.00026 for the dry exhaled breath) and $d$ is the thickness of the air cavity layer [17].

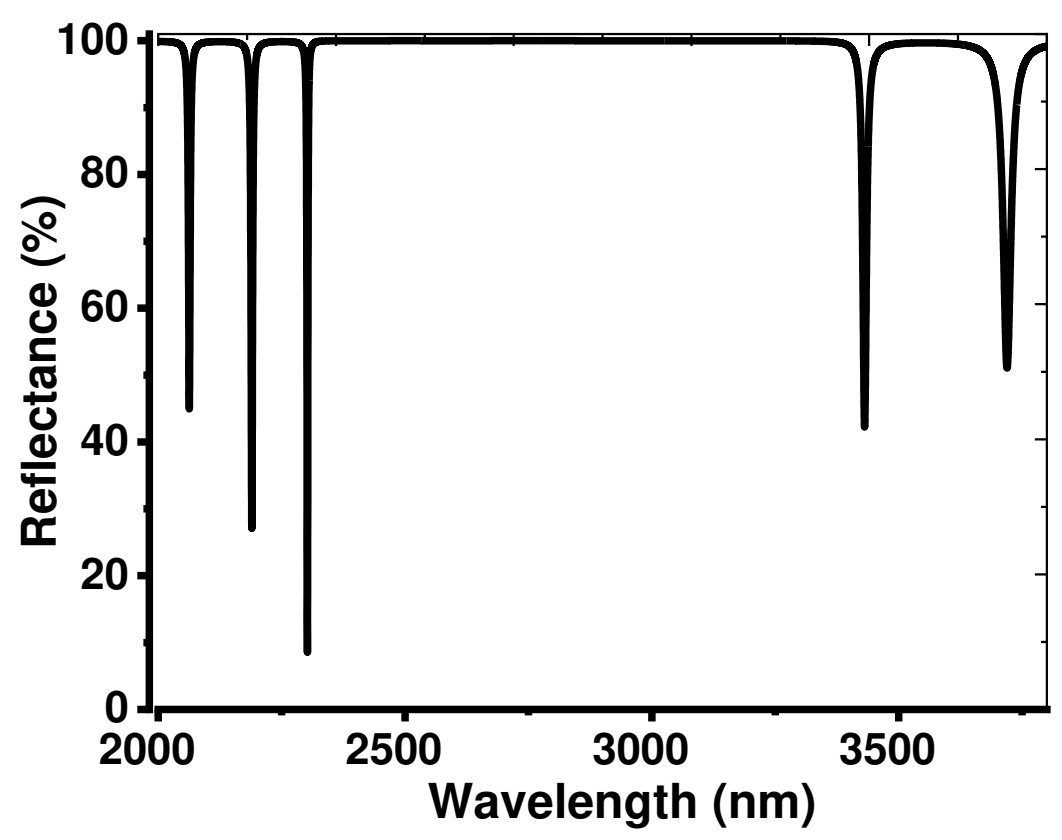

Fig. 3. The reflectance of the $1 D$ PC sensor constructed as prism/air cavity/( $\left.\mathrm{GaN} / \mathrm{SiO}_{2}\right)^{10}$.

Now, a metallic layer from Au with a thickness of $10 \mathrm{~nm}$ is attached on the front of the cavity layer, so the PC structure will be in the new construction as, [prism/Au/air cavity/(GaN/SiO 2$)^{10} /$ air]. As seen in Fig. 4, the resonant dip became more sharper and it was displaced to a new wavelength of $2623.1 \mathrm{~nm}$ with a reflection intensity of $19.55 \%$. This resonant mode represents here the TP resonance due to the presence of the Au layer on the surface of the dielectric 
multilayer stack of [air cavity/GaN/SiO 2$][13,16]$. Besides, the reflection intensity of the ripples outside the bandgap begins to decrease significantly because of the insertion of the Au layer. In other words, the insertion of a metallic layer leads to the increment of the absorption intensity of the EM waves through the PC structure [23]. The reflection intensity, bandwidth, and wavelength value of TP mode are greatly affected with any little change in the parameters of the adjacent layer (air cavity) $[15,16]$. This is the principle of the design isoprene senor.

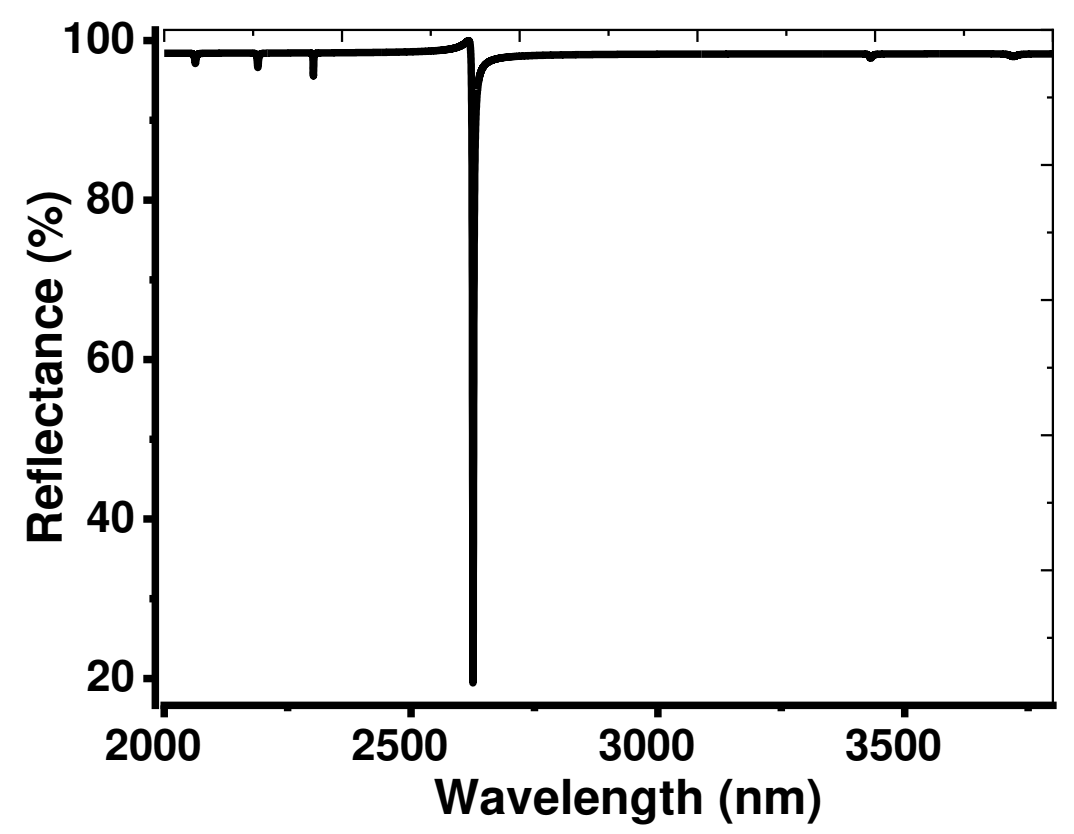

Fig. 4. The reflectance of the ID PC sensor constructed as prism/Au/air cavity/( $\left.\mathrm{GaN} / \mathrm{SiO}_{2}\right)^{10} /$ air.

In the next calculations, we investigate the effect of increasing isoprene levels in the exhaled air components on TP resonance mode and the performance of our designed gas 1D PC sensor [prism/Au/air cavity $/\left(\mathrm{GaN} / \mathrm{SiO}_{2}\right)^{10} / \mathrm{air}$ ]. As mentioned before, the dry exhaled gas of the patient is purposed to pass fill the air cavity layer. In fact, major VOCs of exhaled breath of a healthy person contains a mixture of different gases with disparate ratios as $\mathrm{Ar}(1 \%$ or $10000 \mathrm{ppm}), \mathrm{CO}_{2}(5 \%$ or 
$50000 \mathrm{ppm}), \mathrm{O}_{2}(6 \%$ or $160000 \mathrm{ppm})$, and $\mathrm{N}_{2}(78 \%$ or $780000 \mathrm{ppm})$. In the case of liver fibrosis disease, the levels of isoprene (ppm) are increased in the exhaled breath of unhealthy persons [5]. The effective refractive index $\left(\mathrm{n}_{\mathrm{eff}}\right)$ of the dry exhaled breath is obtained by the rule of the mixture as [32]:

$$
\mathrm{n}_{\mathrm{eff}}^{2}=\mathrm{n}_{1}^{2} \phi_{1}+\mathrm{n}_{2}^{2} \phi_{2}+\mathrm{n}_{3}^{2} \phi_{3}+\cdots
$$

where, $\mathrm{n}$ and $\phi$ represent the index of refraction and volume fraction of each exhaled air gas component. The detailed methods of the refractive index calculation for these gases are described in reference [33, 34]. Meanwhile, we plot in Fig. 5 the effective refractive index of the exhaled breath as a function of isoprene concentrations in the range $0-100$ ppm. As shown in Fig. 5 (a) and (b), the effective refractive index of the air cavity is roughly increasing with the increasing of the concentrations or levels of isoprene. The effective refractive index has increased from 1.00026142 to 1.00037811 due to the concentration of isoprene variation from $0 \mathrm{ppm}$ to $100 \mathrm{ppm}$. We could see that the amount of increment of the effective refractive index with isoprene levels is considered very small which represents a big challenge to any sensor or biomarker to sense this tiny change in the gas concentration or its relevant refractive index. However, we will see that our proposed 1D PC sensor can sense these very low concentrations accurately and with high sensitivity, which might be used in the nearest future for designing a sensitive PC sensor for such very low gas concentrations. In addition to that, the effective refractive index is approximately unchanged with the wavelength, which gives the sensor an additional advantage in the stability of calculations versus different wavelengths. 

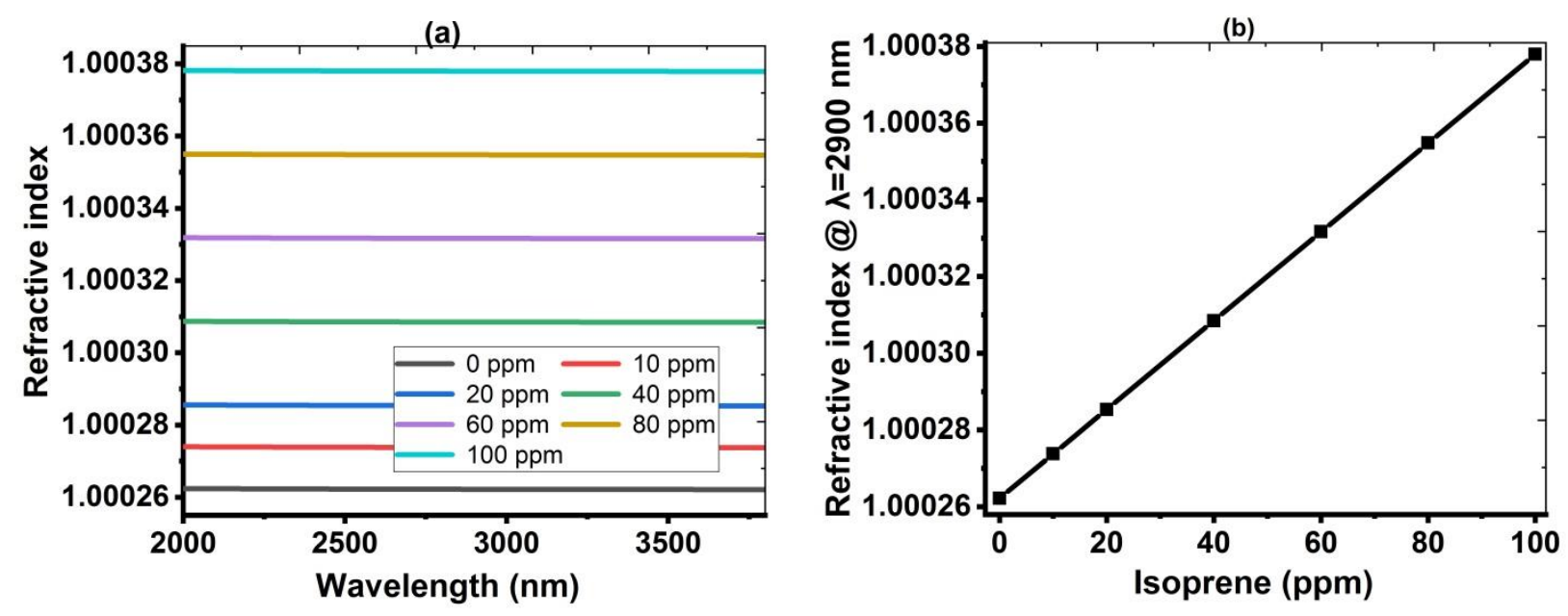

Fig. 5. (a) The effective refractive index of the air cavity versus isoprene concentrations (in ppm) and wavelength of the incident EM waves, (b) the relation between the effective refractive index and isoprene concentrations at $\lambda=2900 \mathrm{~nm}$.

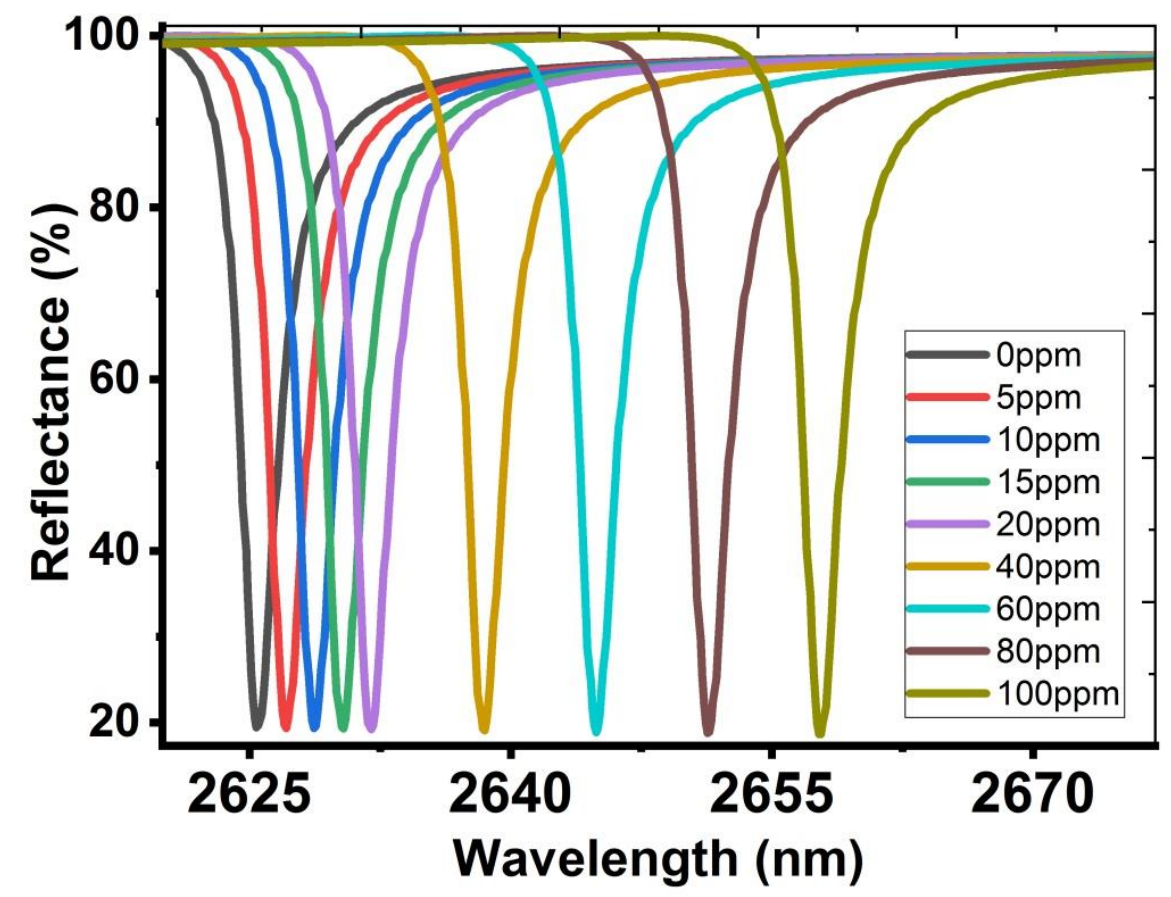

Fig. 6. The Reflection spectrum of the ID PC gas sensor [prism/Au/air cavity/( $\left(\mathrm{GaN}_{\mathrm{SiO}}\right)^{10}{ }^{10}$ at different isoprene concentrations from 0 to $100 \mathrm{ppm}$. 
Now, we will identify the position of the TP resonance mode and reflection spectrum of the PC design, [prism/Au/air cavity/(GaN/SiO 2$)^{10} /$ air] at different isoprene levels. As shown in Fig. 6, the TP resonance mode is displaced towards longer wavelengths with increasing isoprene concentrations from $0 \mathrm{ppm}$ to 100 ppm. The TP resonance mode shifted from 2625.3 to 2627.1 due to the increase of isoprene concentration to the value of $5 \mathrm{ppm}$. For higher concentrations up to 10 , $15,20,40,60,80$, and $100 \mathrm{ppm}$, the TP resonance mode is moved towards the wavelengths 2628.7, 2630.4, 2632, 2638.51, 2644.9, 2651.29, and $2657.69 \mathrm{~nm}$, respectively. Hence, the TP resonance mode is moved about $32.39 \mathrm{~nm}$ for a just concentration increase of $100 \mathrm{ppm}$. Such a result may give us an excellent impression about the sensitivity of the prosed gas sensor and how it can sense these lower concentrations of any gas with higher sensitivity.

Depending on the results of Fig. 6 we have plotted in Fig. 7 the dependence of the TP resonance wavelength position on isoprene concentration. The figure concluded that that, the wavelength position of the TP resonance mode directly increases with increasing isoprene concentrations. Such relationship can be obtained in an equation form by taking the linear fitting of Fig.7 as,

$$
\lambda_{T P}=2625.54+0.321 C \text {. }
$$

Where, $\mathrm{C}$ is the isoprene concentration in ppm. From that figure and the above equation, we could obtain the sensitivity of the proposed 1D PC gas sensor by taking the slope of the relation in Fig.7 [35 - 37]. Herein, we could obtain higher sensitivity that reaches the value of $0.321 \mathrm{~nm} / \mathrm{ppm}$ or $278720 \mathrm{~nm} / \mathrm{RIU}$. Consequently, this sensitivity value is considered very high compared with other PC counterparts $[13,21]$. 


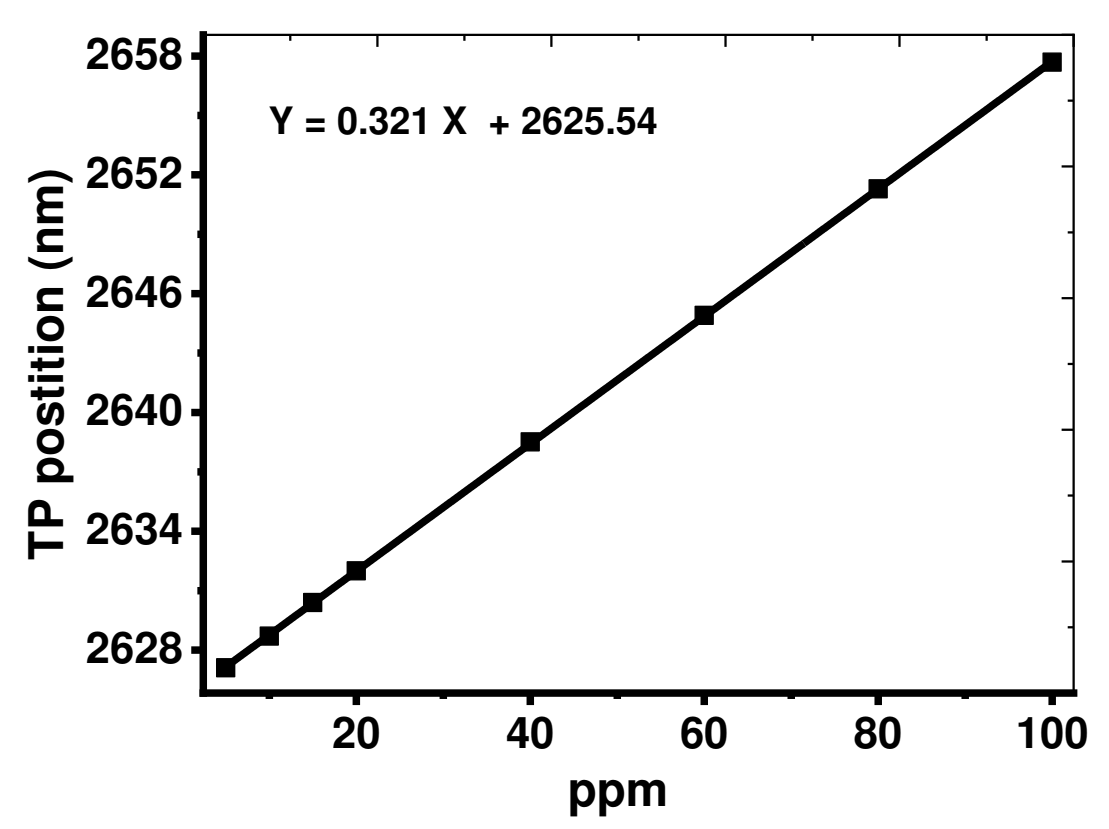

Fig. 7. The wavelength position of the TP resonance mode as a function of isoprene levels.

The important parameters that determine the performance of any sensor was calculated for the proposed sensor. These parameters include detection limit (DL), figure of merit (FoM), sensitivity (S), quality factor (QF), detection accuracy (DA), signal to noise ratio (SNR), dynamic range (DR), and sensor resolution (SR). The values of these performance parameters based on the different isoprene concentrations are summarized in table (1). Also, their mathematical expressions can be written in the following equations [38 - 42],

$$
\begin{gathered}
Q F=\frac{\lambda_{\text {res }}}{F W H M}, \\
S=\frac{\Delta \lambda}{\Delta C},
\end{gathered}
$$




$$
\begin{gathered}
S N R=\frac{\Delta \lambda}{F W H M}, \\
D L=\left(\frac{1}{S}\right) \frac{F W H M}{1.5(S N R)^{0.25}}, \\
S R=D L S,
\end{gathered}
$$

Where, $\lambda_{\text {res }}$ is the wavelength position of the TP resonance dip, FWHM is the full width at half maximum of the TP resonant wavelength. The isoprene concentration of $0 \mathrm{ppm}$ is taken as the reference concentration for all calculations.

The results obtained in table (1) show that our 1D PC gas sensor achieved not only high sensitivity but also high performance based on the other performance parameters. The value of the reduced sensitivity or the so-called FoM reached higher than $0.134 / \mathrm{ppm}$. Besides the stability of the reflection intensity for all isoprene levels, it did not decrease than $18 \%$ in the worst case. In addition to that, the detection accuracy and detection limit of the sensor reached higher than 0.37 and $5.45 \mathrm{ppm}$, respectively. Moreover, the sharpness of each TP resonance dip is very brilliant represented by the high QF (more than 985) and low FWHM (not more than $2.7 \mathrm{~nm}$ for all TP dips). 
Table (1):- The performance of our designed 1D PC gas sensor as a function of isoprene concentrations.

\begin{tabular}{|c|c|c|c|c|c|c|c|c|c|}
\hline ppm & $\mathbf{0}$ & 5 & 10 & 15 & 20 & 40 & 60 & 80 & 100 \\
\hline position (nm) & 2625.3 & 2627.1 & 2628.7 & 2630.4 & 2632 & 2638.51 & 2644.9 & 2651.29 & 2657.69 \\
\hline $\mathbf{S}(\mathbf{n m} / \mathbf{p p m})$ & - & 0.36 & 0.34 & 0.34 & 0.335 & 0.330 & 0.326 & 0.324 & 0.323 \\
\hline FWHM (nm) & 2.68 & 2.67 & 2.69 & 2.67 & 2.68 & 2.69 & 2.7 & 2.7 & 2.7 \\
\hline Intensity (\%) & 19.45 & 19.36 & 19.33 & 19.31 & 19.21 & 19.09 & 18.87 & 18.77 & 18.66 \\
\hline SNR & - & 0.674 & 1.263 & 1.910 & 2.5 & 4.910 & 7.259 & 9.625 & 11.99 \\
\hline FoM (/ ppm) & - & 0.134 & 0.126 & 0.127 & 0.125 & 0.122 & 0.120 & 0.1203 & 0.1199 \\
\hline $\mathbf{Q F}$ & 979.5 & 983.9 & 977.2 & 985.1685 & 982.08 & 980.8 & 979.5 & 981.9 & 984.3 \\
\hline DA (/nm) & 0.3731 & 0.3745 & 0.3717 & 0.3745 & 0.3731 & 0.3717 & 0.3703 & 0.3703 & 0.3703 \\
\hline DR & 1603.6 & 1607.75 & 1602.74 & 1609.77 & 1607.75 & 1608.72 & 1609.6 & 1613.52 & 1617.41 \\
\hline DL (ppm) & - & 5.45 & 4.97 & 4.45 & 4.24 & 3.64 & 3.35 & 3.145 & 2.98 \\
\hline SR (nm) & - & 0.906 & 1.060 & 1.175 & 1.257 & 1.488 & 1.641 & 1.761 & 1.861 \\
\hline
\end{tabular}

Finally, table (2) shows the advantage of our sensor over previous optical sensors by its higher sensitivity. This study has focused on a novel design of a 1D PC isoprene sensor dependent on the optical TP state in dry exhaled breath for liver fibrosis diagnosis. The proposed gas sensor is constructed as, [prism/Au/air cavity $/\left(\mathrm{GaN} / \mathrm{SiO}_{2}\right)^{10} /$ air]. $\mathrm{GaN}$ layer is set with a thickness $=200 \mathrm{~nm}, \mathrm{SiO}_{2}$ layer is set with a thickness $=900 \mathrm{~nm}$, the number of periods is proposed to be $\mathrm{N}=10$, the angle of incidence is taken as $\theta_{0}=50^{\circ}$ and the prism is assumed with the index $n_{p}=$ 1.3. The transfer matrix method is used to calculate the reflection spectra of the incident EM waves and the rule of the mixture is adopted for the calculation of refractive indices of gases. The results have found that the TP resonance 
wavelength position is highly sensitive to the smallest change in isoprene concentrations in ppm. Moreover, our proposed gas sensor provided higher sensitivity of $0.321 \mathrm{~nm} / \mathrm{ppm}$ or $278720 \mathrm{~nm} / \mathrm{RIU}$ and a QF of 985 . Finally, the imposed gas sensor here could be of potential interest for monitoring several diseases based on the type and level of the exogenous VOC occurring in the exhaled breath.

Table (2):- A comparison between our sensor sensitivity and some relevant previous sensors.

\begin{tabular}{|c|c|}
\hline Reference & Sensitivity $(\mathrm{nm} /$ RIU) \\
\hline$[13]$ & 5018 \\
\hline$[21]$ & 575 \\
\hline$[43]$ & 850 \\
\hline$[44]$ & 70 \\
\hline$[45]$ & 970 \\
\hline$[46]$ & 450 \\
\hline$[47]$ & 9615 \\
\hline$[48]$ & 1179 \\
\hline$[49]$ & 190000 \\
\hline Our design & 278720 \\
\hline
\end{tabular}

\section{Conclusion}

This study has focused on a novel design of a 1D PC isoprene sensor dependent on the optical TP state in dry exhaled breath for liver fibrosis diagnosis. The proposed gas sensor is constructed as, [prism/Au/air cavity/(GaN/ SiO2)10/air]. GaN layer is 
set with a thickness $=200 \mathrm{~nm}, \mathrm{SiO} 2$ layer is set with a thickness $=900 \mathrm{~nm}$, the number of periods is proposed to be $\mathrm{N}=10$, the angle of incidence is taken as $\theta_{\mathrm{o}}=50^{\circ}$ and the prism is assumed with the index $\mathrm{np}=1.3$. The transfer matrix method is used to calculate the reflection spectra of the incident EM waves and the rule of the mixture is adopted for the calculation of refractive indices of gases. The results have found that the TP resonance wavelength position is highly sensitive to the smallest change in isoprene concentrations in ppm. Moreover, our proposed gas sensor provided higher sensitivity of $0.321 \mathrm{~nm} / \mathrm{ppm}$ or $278720 \mathrm{~nm} / \mathrm{RIU}$ and a QF of 985. Finally, the imposed gas sensor here could be of potential interest for monitoring several diseases based on the type and level of the exogenous VOC occurring in the exhaled breath.

Author contribution:- (1) The authors made substantial contributions to the conception and design, and / or acquisition of data, and / or analysis and interpretation of data. (2) The authors participated in drafting the article or revising it critically for important intellectual content. (3) The authors gave the final approval of the version to be submitted. All the authors contributed equally.

Funding:- Not applicable

Availability of Data and Material:- The data that support the findings of this study are available from the corresponding author upon reason-able request.

Code availability:- Not applicable

Ethics approval:- I, hereby, the corresponding author declare that the authors have thoroughly read the Journal Policy and admitted all its requirements. Specifically, I declare here that this contribution is original and has not been published anywhere. 
I also declare that this article doesn't contain any plagiarized materials. No part of this manuscript has been introduced in any conference or published in any journal.

\section{Declarations:-}

Consent to participate:- All authors accepted.

Consent for publication:- All authors accepted.

Competing interests:- The authors declare no competing interests.

\section{References}

[1] H. Schwoebel, R. Schubert, M. Sklorz, S. Kischkel, R. Zimmermann, J. K. Schubert, W. Miekisch, Phase-Resolved Real-time Breath Analysis during Exercise by means of Smart Processing of PTR-MS Data, Anal. Bioanal. Chem.401 (7) (2011) 2079 - 2091. https://doi.org/10.1007/s00216-011-5173-2

[2] L. Pauling, A. B. Robinson, R. Teranishi, P. Cary, Quantitative analysis of urine vapor and breath by gas-liquid partition chromatography, Proc. Natl. Acad. Sci. USA, 68 (1971) 2374-2376. https://doi.org/10.1073/pnas.68.10.2374

[3] M. Phillips, Breath test in medicine, Sci. Am. 267 (1992) 74-79. https://doi.org/10.1038/scientificamerican0792-74

[4] A. H. Jalal, F. Alam, S. Roychoudhury, Y. Umasankar, N. Pala, S. Bhansali, Prospects and Challenges of Volatile Organic Compound Sensors in Human Healthcare, ACS Sensors, 3(7) (2018) $1246 \quad-1263$. https://doi.org/10.1021/acssensors.8b00400

[5] C. Hayton, D. Terrington, A. M. Wilson, N. Chaudhuri, C. Leonard, S. J. Fowler, Breath biomarkers in idiopathic pulmonary fibrosis: a systematic review, Respiratory Research, 20(1) (2019) 7. https://doi.org/10.1186/s12931-019-0971-8

[6] A. Tangerman, M. T. Meuwese-Arends, J. H. van Tongeren, New Sensitive Assay for Measuring Volatile Sulphur Compounds in Human Breath by Tenax 
Trapping and Gas Chromatography and Its Application in Liver Cirrhosis, Clin. Chim. Acta. 130 (1) (1983) 103-110. https://doi.org/10.1016/0009$\underline{8981(83) 90263-2}$

[7] C. Popa, M. Petrus, A. M. Bratu, Ammonia and ethylene biomarkers in the respiration of the people with schizophrenia using photoacoustic spectroscopy, Journal of Biomedical Optics, 20(5) (2015) 057006. https://doi.org/10.1117/1.JBO.20.5.057006

[8] J. E. Szulejko, M. McCulloch, J. Jackson, L. D. McKee, J. C. Walker, T. Solouki, Evidence for Cancer Biomarkers in Exhaled Breath, IEEE Sensors Journal, 10(1) (2010) 185-210. https://doi.org/10.1109/JSEN.2009.2035669

[9] J. Li, T. M. Smeeton, M. Zanola, J. Barrett, V. Berryman-Bousquet, A compact breath acetone analyzer based on an ultraviolot light emitting diode, Sens. Actuators B Chem., 273 (2018) 76-82. https://doi.org/10.1016/j.snb.2018.05.114 [10] L. Gao, X. Yang, X. Chen, J. Wang, Ionic liquid-based slab optical waveguide sensor for the detection of ammonia in human breath, J. Colloid Interface Sci., 512 (2018) 819-825. https://doi.org/10.1016/j.jcis.2017.10.114

[11] A. M. Bratu, M. Petrus, C. Popa, Laser-based spectrometer for opitcal trace gas detection in young adults with autism, Microchem. J., 138 (2018) 203-208. https://doi.org/10.1016/j.microc.2018.01.014

[12] H. A. Elsayed, A. Mehaney, A new method for glucose detection using the one dimensional defective photonic crystals, Materials Research Express, 6 (2019) 036201. https://doi.org/10.1088/2053-1591/aaf3da

[13] A. M. Ahmed, A. Mehaney, Ultra-high sensitive 1D porous silicon photonic crystal sensor based on the coupling of Tamm/Fano resonances in the mid-infrared region, Scientific Reports, 9 (2019) 6973. https://doi.org/10.1038/s41598-01943440-y 
[14] A. Chiappini, L. T. N. Tran, P. M. Trejo-García, L. Zur, A. Lukowiak, M. Ferrari, G. C. Righini, Photonic crystal stimuli-responsive chromatic sensors: A $\begin{array}{lllll}\text { short } & \text { review, } & \text { Micromachines, } & \text { 11(3) }\end{array}$ https://doi.org/10.3390/mi11030290

[15] H. Elsayed, A. Mehaney, Theoretical verification of photonic crystals sensor for biodiesel detection and sensing, Physica Scripta, 95 (2020) 085507. https://doi.org/10.1088/1402-4896/aba2b1

[16] A. M. Ahmed, A. Mehaney, Novel design of wide temperature ranges sensor based on Tamm state in a pyroelectric photonic crystal with high sensitivity, Physica E, 125 (2020) 114387. https://doi.org/10.1016/j.physe.2020.114387

[17] M. M. Abadla, H. A. Elsayed, A. Mehaney, Thermo-optical properties of binary one dimensional annular photonic crystal including temperature dependent $\begin{array}{llllll}\text { constituents, } & \text { Physica } & \text { E, } & 119 & \text { (2020) } & 114020 .\end{array}$ https://doi.org/10.1016/j.physe.2020.114020

[18] J. Haberko, L. S. Froufe-Pérez, F. Scheffold, Transition from light diffusion to localization in three-dimensional amorphous dielectric networks near the band edge, Nature $\quad$ Communications, 11(1) $\quad$ (2020) 4867. https://doi.org/10.5281/zenodo.3968424

[19] M. Shaban, A. M. Ahmed, E. Abdel-Rahman, H. Hamdy, Tunability and sensing properties of plasmonic/1D photonic crystal, Scientific Reports, 7(1) (2017) 41983. https://doi.org/10.1038/srep41983

[20] H. Clevenson, P. Desjardins, X. Gan, D. Englund, High sensitivity gas sensor based on high-Q suspended polymer photonic crystal nanocavity, Applied Physics Letters, 104(24) (2014) 241108. https://doi.org/10.1063/1.4879735

[21] Y.-H. Chen, W.-H. Shi, L. Feng, X.-Y. Xu, M.-Y. Shang-Guan, Study on simultaneous sensing of gas concentration and temperature in one dimensional 
photonic crystal, Superlattices and Microstructures, 131 (2019) 53-58. https://doi.org/10.1016/j.spmi.2019.05.033

[22] A. Anamoradi, K. Fasihi, A highly sensitive optofluidic-gas sensor using two dimensional photonic crystals, Superlattices and Microstructures, 125 (2019) 302309. https://doi.org/10.1016/j.spmi.2018.11.019

[23] H.-P. Chiang, H.-T. Yeh. C.-M. Chen, J.-C. Wu, S.-Y. Su, R. Chang, Y.-J. Wu, D. P. Tsai, P. T. Leung, Surface plasmon resonance monitoring of temperature via phase measurement, Opt. Commun., 241 (2004) 409-418. https://doi.org/10.1016/j.optcom.2004.07.045

[24] B. Auguie, M. C. Fuertes, P. C. Angelome, N. L. Abdala, S. G. J. A. A. Illia, A. Fainstein, Tamm plasmon resonance in mesoporous multilayers: toward a sensing application, ACS Photonics, 1(9) (2014) 775-780. https://doi.org/10.1021/ph5001549

[25] L. Simurka, R. Ctvrtlik, J. Tomastik, G. Bektas, J. Svoboda, and K. Bange, Mechanical and optical properties of $\mathrm{SiO}_{2}$ thin films deposited on glass, Chem. Pap., 72 (2018) 2143-2151. https://doi.org/10.1007/s11696-018-0420-Z

[26] C. H. Hsu, L. H. Chien, P. L. Kuo, High thermal and electrochemical stability of a $\mathrm{SiO} 2$ nanoparticle hybird-polyether cross-linked membrane for safety reinforced lithium-ion batteries, RSC Adv., 6 (2016) 18089-18095. https://doi.org/10.1039/C5RA26694G

[27] A. H. Aly, D. Mohamed, H. A. Elsayed, A. Mehaney, Fano resonance by means of the one-dimensional superconductor photonic crystals, J. Supercond. Nov. Magn., 31 (2018) 3827-3833. https://doi.org/10.1007/s10948-018-4660-5 [28] M. N. Armenise, C. E. Campanella, C. Ciminelli, F. Dell'Olio, and V. M. N. Passaro, Phononic and photonic band gap structures: Modelling and applications, Phys. Procedia 3, (2010) 357-364. https://doi.org/10.1016/j.phpro.2010.01.047 
[29] A. Alabastri, S. Tuccio, A. Giugni, A. Toma, C. Liberale, G. Das, F. De Angelis, E. Di Fabrizio, R. Zaccaria, Molding of plasmonic resonances in metallic nanostructures: dependence of the non-linear electric permittivity on system size and temperature, Mater., 6 (2013) 4879-4910. https://doi.org/10.3390/ma6114879.

[30] , I. H. Malitson, Interspecimen Comparison of the Refractive Index of Fused Silica, Journal of the Optical Society of America, 55 (1965) 1205. https://doi.org/10.1364/JOSA.55.001205

[31] A. S.Barker, M. Ilegems, Infrared Lattice Vibrations and Free-Electron Dispersion in GaN, Physical Review B, 7(2) (1973) 743-750. https://doi.org/10.1103/PhysRevB.7.743

[32] M. Li, Y. Song, High effective sensors based on photonic crystals, Frontiers of Chemistry in China, 5(2) (2010) 115 - 122. https://doi.org/10.1007/s11458-010$\underline{0104-\mathrm{X}}$

[33] J. Zhang, Z. H. Lu, L. J. Wang, Precision refractive index measurements of air, $\mathrm{N}_{2}, \mathrm{O}_{2}, \mathrm{Ar}$, and $\mathrm{CO}_{2}$ with a frequency comb, Applied Optics, 47(17) (2008) 3143 - 3151. https://doi.org/10.1364/AO.47.003143

[34] E. M. Pearce, Kirk-Othmer, encyclopedia of chemical technology, 3rd ed., Vol. I, Wiley-Interscience, New York, 1978.

[35] I. M. White, X. Fan, On the performance quantification of resonant refractive $\begin{array}{llllll}\text { index } & \text { sensors, } & \text { Opt. } & \text { Express, } & 16 & \text { (2008) }\end{array}$ https://doi.org/10.1364/OE.16.001020

[36] M. El Beheiry, V. Liu, S. Fan, O. Levi, Sensitivity enhancement in photonic crystal slab biosensors, Opt. Express, $18 \quad$ (22) (2010) 22702. https://doi.org/10.1364/OE.18.022702

[37] D. K. C. Wu, K. J. Lee, V. Pureur, and B. T. Kuhlmey, Performance of refractive index sensors based on directional couplers in photonic crystal fibers, $\mathrm{J}$. 
Light. Technol., $\quad 31 \quad$ (22) $\quad$ (2013) $3500 \quad$ - 3510. https://doi.10.1109/JLT.2013.2283496.

[38] I. M. White and X. Fan, On the performance quantification of resonant refractive index sensors, Opt. Express, 16(2) (2008) 1020. https://doi.org/10.1364/OE.16.001020

[39] M. El Beheiry, V. Liu, S. Fan, and O. Levi, Sensitivity enhancement in photonic crystal slab biosensors, Opt. Express, 18(22) (2010) 22702. https://doi.org/10.1364/OE.18.022702

[40] L. Rindorf, J. B. Jensen, M. Dufva, L. H. Pedersen, P. E. Hoiby, O. Bang, Photonic crystal fiber long period gratings for biochemical sensing, Opt. Exp., 14(2006) 8224-8231. https://doi.org/10.1364/OE.14.008224

[41] M. Naftaly and R. Dudley, Methodologies for determining the dynamic ranges and signal-to-noise ratios of terahertz time-domain spectrometers, Opt. Lett., 34(8) (2009) 1213. https://doi.org/10.1364/OL.34.001213

[42] A. M. Ahmed, M. Shaban, Highly sensitive $\mathrm{Au}-\mathrm{Fe} 2 \mathrm{O} 3-\mathrm{Au}$ and $\mathrm{Fe} 2 \mathrm{O} 3-\mathrm{Au}-$ Fe2O3 biosensors utilizing strong surface plasmon resonance, Appl. Phys. B Lasers Opt., 126(4) (2020) 1 - 10. https://doi.org/10.1007/s00340-020-7405-7

[43] X. Huang, J. Yang, C. Tao, X. Guo, H. Bao, Y. Yin, H. Chen, Y. Zhu, An inline Mach-Zehnder interferometer using thin-core fiber for ammonia gas sensing with high sensitivity, Scientific reports, $7 \quad$ (2017) 44994. https://doi.org/10.1038/srep44994

[44] M. C. Sansierra, J. Morrone, F. Cornacchiulo, M. C. Fuertes, P. C. Angelomé, Detection of Organic Vapors Using Tamm Mode Based Devices Built from Mesoporous Oxide Thin Films, Chem. Nano. Mat., 5 (2019) 1289 - 1295. https://doi.org/10.1002/cnma.201900388 
[45] R. Das, T. Srivastava, R. Jha, On the performance of Tamm-plasmon and surface-plasmon hybrid-mode refractive-index sensor in metallo-dielectric heterostructure configuration, Sensors and Actuators B: Chemical, 206 (2015) 443 - 448. https://doi.org/10.1016/j.snb.2014.09.032

[46] X. Qian, Y. Zhao, Y.-n. Zhang, Q. Wang, Theoretical research of gas sensing method based on photonic crystal cavity and fiber loop ring-down technique, Sensors and Actuators B: Chemical, 228 (2016) 665 - 672. https://doi.org/10.1016/j.snb.2016.01.087

[47] S. Sharma, V. S. Chaudhary, D. Kumar, Design of chemical sensor based on dual core photonic crystal fiber, Materials Today: Proceedings, 33(5) (2020) 2122 -2124. https://doi.org/10.1016/j.matpr.2020.02.889

[48] Y. Chen, J. Dong, T. Liu, Q. Zhu, W. Chen, Refractive index sensing performance analysis of photonic crystal containing graphene based on optical Tamm state, Modern Physics Letters B, $30 \quad$ (2016) 1650030. https://doi.org/10.1142/S0217984916500305

[49] Z. A. Zaky, A. M. Ahmed, A. S. Shalaby, A. H. Aly, Refractive index gas sensor based on the Tamm state in a one-dimensional photonic crystal: Theoretical optimization, $\quad$ Scientific $\quad$ Reports, $10(1) \quad$ (2020) 9736. https://doi.org/10.1038/s41598-020-66427-6

\section{Conflicts of Interest}

The authors declare they have no conflicts of interest. 
Figures

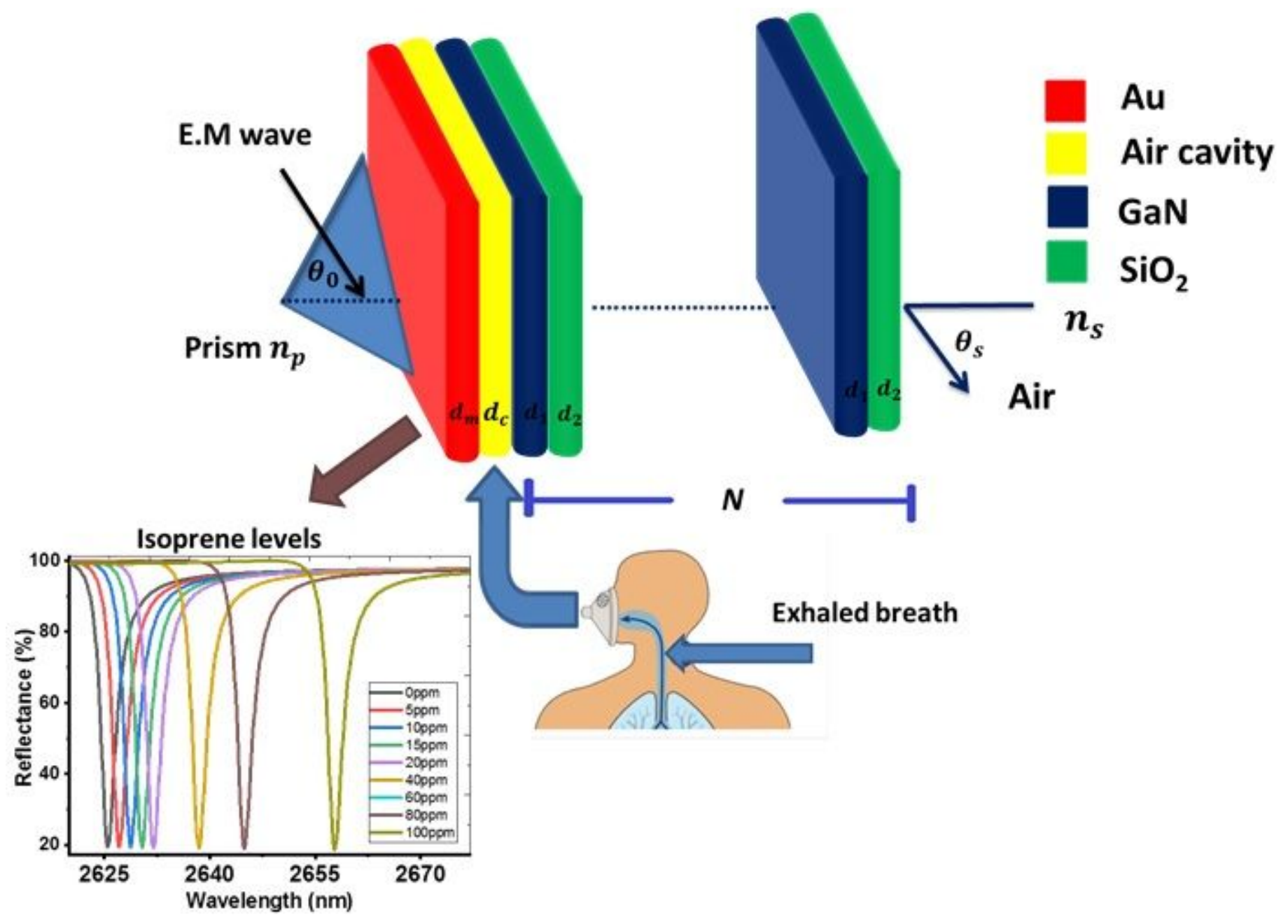

Figure 1

The schematic diagram of the proposed sensor design [prism/Au/air cavity/(GaN/SiO2)N]. 


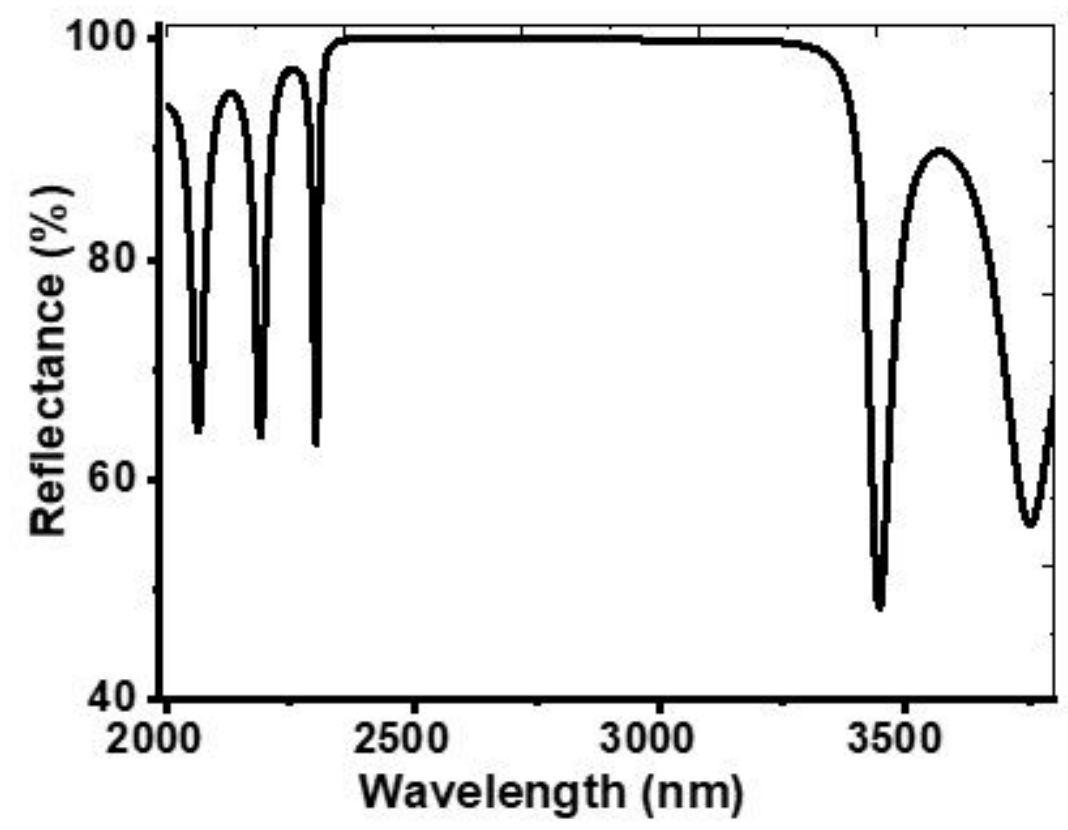

Figure 2

The reflectance of the 1D PC gas sensor that constructed as prism/(GaN/SiO2)10/air.

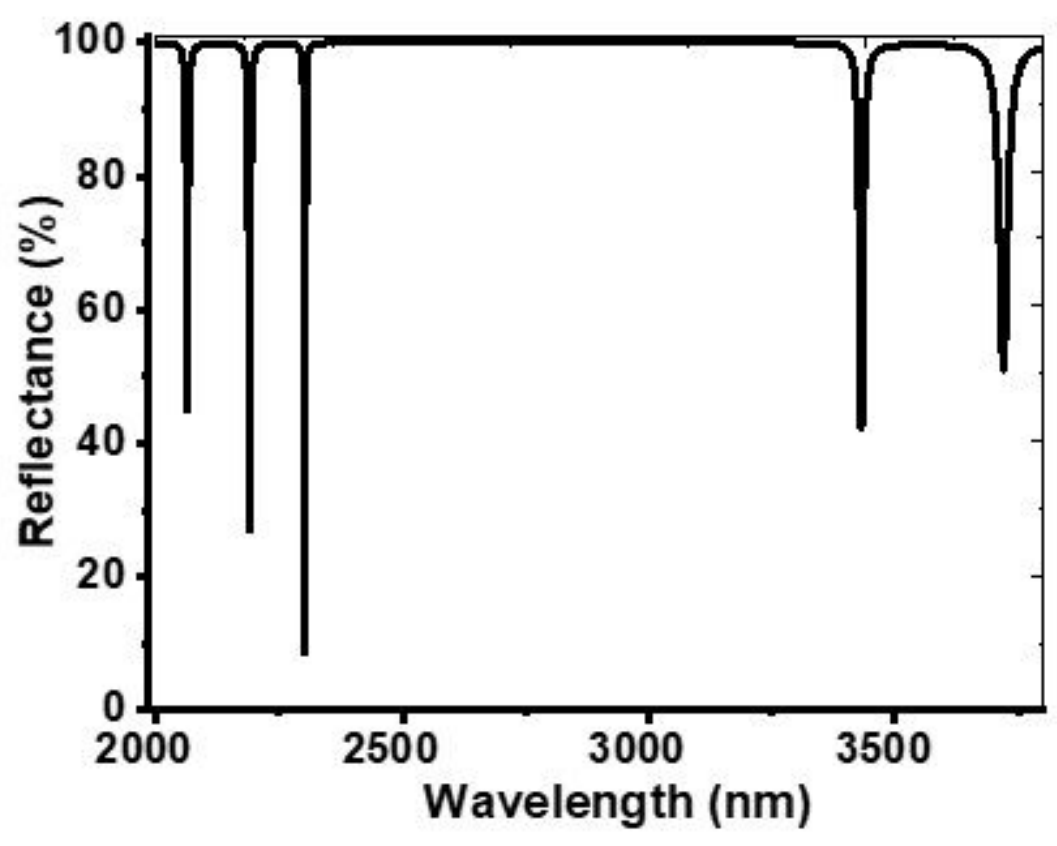

Figure 3

The reflectance of the 1D PC sensor constructed as prism/air cavity/(GaN/SiO2)10. 


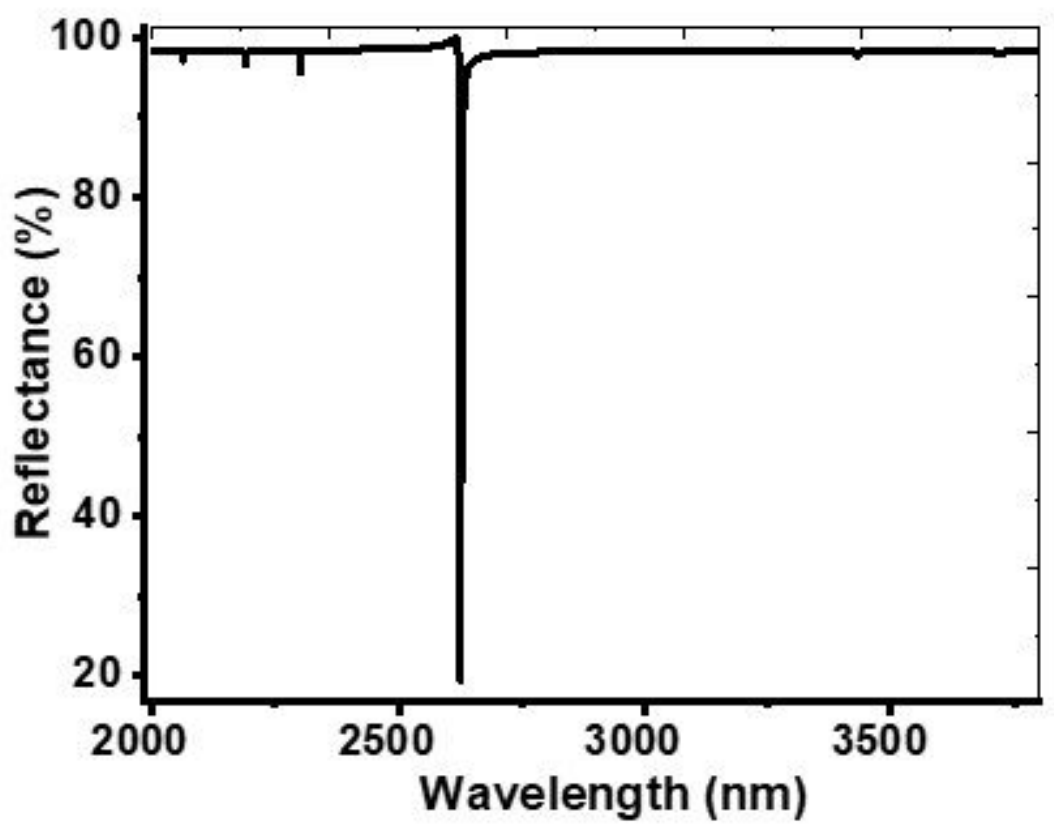

Figure 4

The reflectance of the 1D PC sensor constructed as prism/Au/air cavity/(GaN/SiO2)10/air.
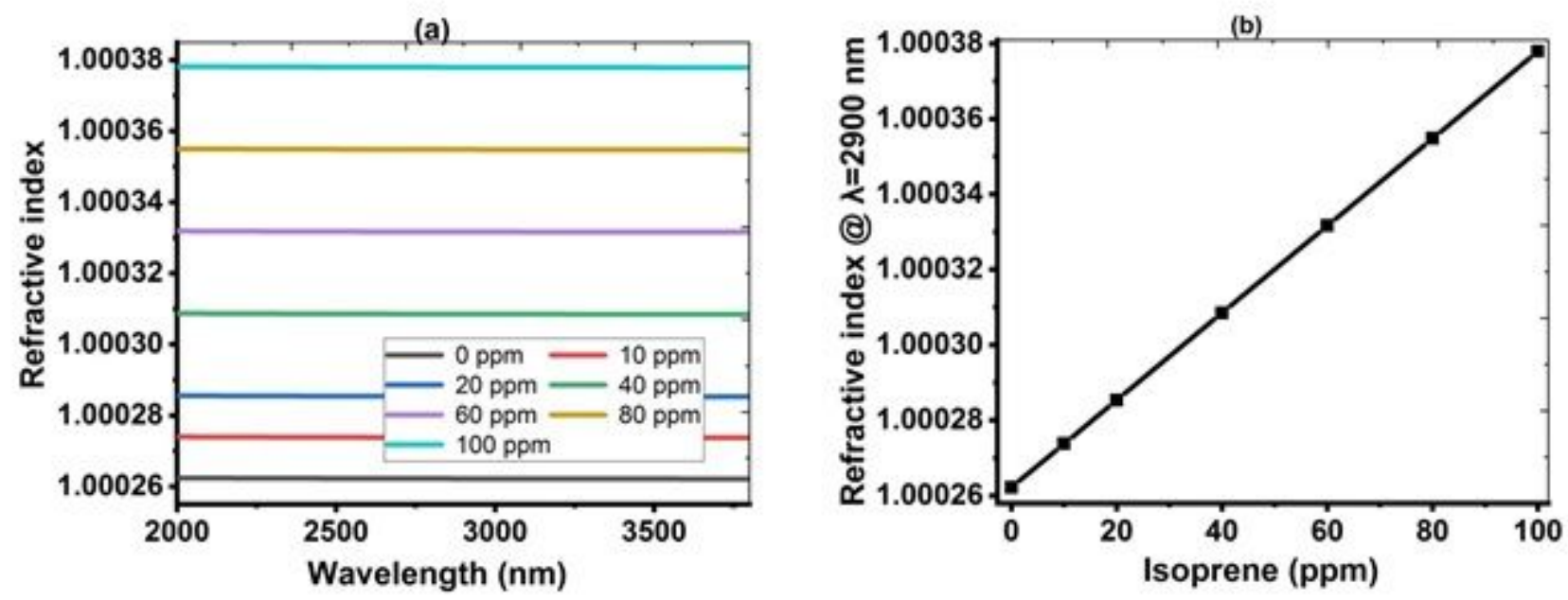

Figure 5

(a) The effective refractive index of the air cavity versus isoprene concentrations (in ppm) and wavelength of the incident EM waves, (b) the relation between the effective refractive index and isoprene concentrations at $\lambda=2900 \mathrm{~nm}$. 


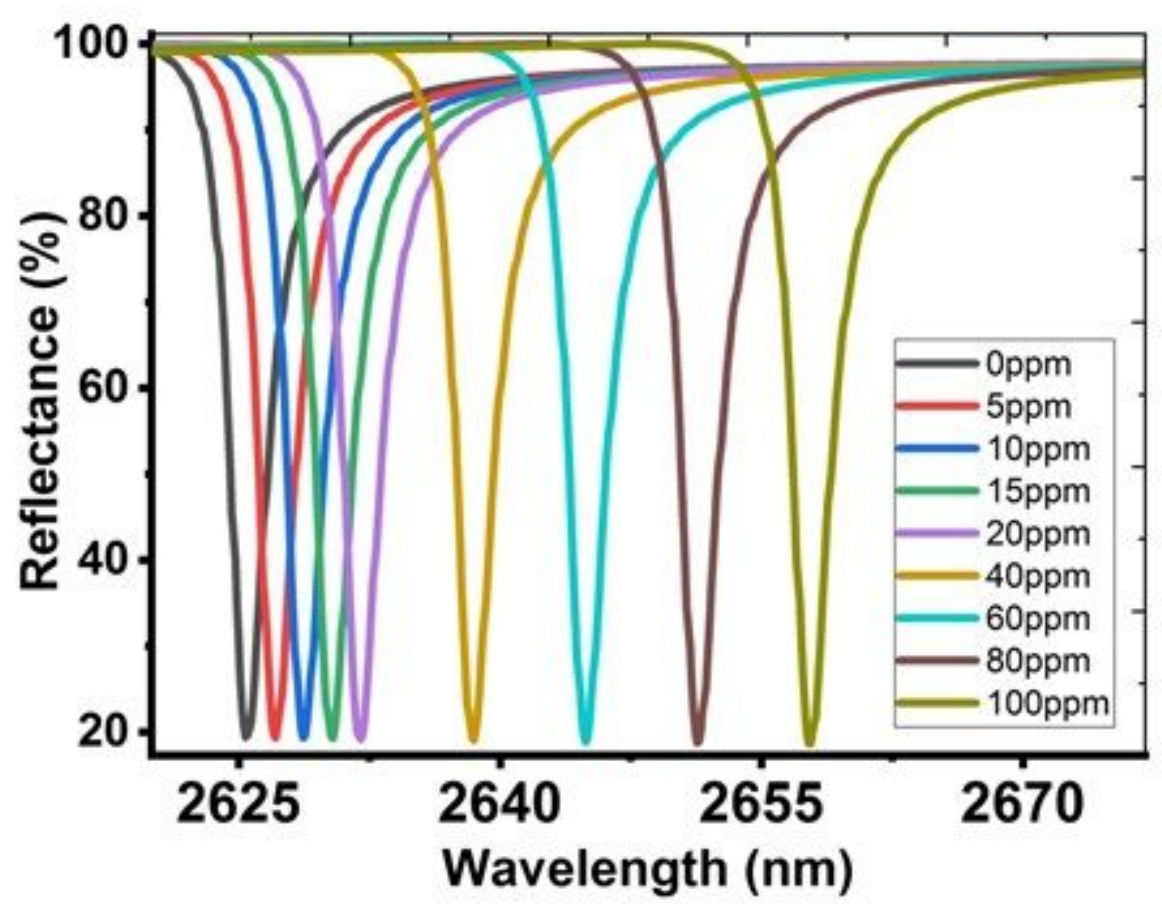

Figure 6

The Reflection spectrum of the 1D PC gas sensor [prism/Au/air cavity/(GaN/SiO2)10] at different isoprene concentrations from 0 to $100 \mathrm{ppm}$.

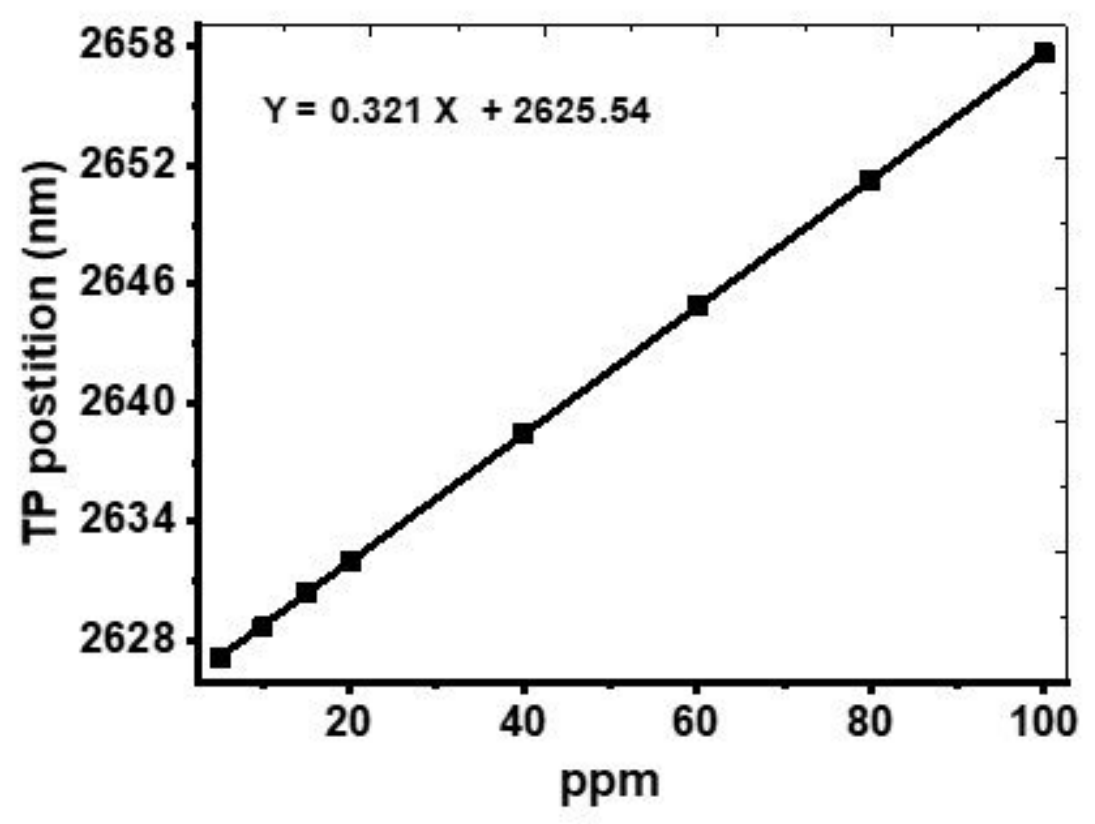

Figure 7

The wavelength position of the TP resonance mode as a function of isoprene levels. 